\title{
Fractal Metrology for biogeosystems analysis
}

\author{
V. Torres-Argüelles ${ }^{1}$, K. Oleschko ${ }^{2}$, A. M. Tarquis ${ }^{3}$, G. Korvin ${ }^{4}$, C. Gaona ${ }^{2}$, J.-F. Parrot ${ }^{5}$, and E. Ventura-Ramos ${ }^{1}$ \\ ${ }^{1}$ Universidad Autónoma de Querétaro, Querétaro, Mexico \\ ${ }^{2}$ Centro de Geociencias, Universidad Nacional Autónoma de México (UNAM), Blvd. Juriquilla 3001, \\ 76220, Querétaro, Mexico \\ ${ }^{3}$ Dept. of Applied Mathematics to Agricultural Engineering (E.T.S.I.A.) C.E.I.G.R.A.M., \\ Technical University of Madrid (U.P.M.), Ciudad Universitaria, 28040, Madrid, Spain \\ ${ }^{4}$ King Fahd University of Petroleum and Minerals, Dhahran 31261, Saudi Arabia \\ ${ }^{5}$ Instituto de Geografía, UNAM, Ciudad Universitaria, México, Distrito Federal, Mexico
}

Received: 15 April 2010 - Published in Biogeosciences Discuss.: 22 June 2010

Revised: 12 October 2010 - Accepted: 12 October 2010 - Published: 24 November 2010

\begin{abstract}
The solid-pore distribution pattern plays an important role in soil functioning being related with the main physical, chemical and biological multiscale and multitemporal processes of this complex system. In the present research, we studied the aggregation process as self-organizing and operating near a critical point. The structural pattern is extracted from the digital images of three soils (Chernozem, Solonetz and "Chocolate" Clay) and compared in terms of roughness of the gray-intensity distribution quantified by several measurement techniques. Special attention was paid to the uncertainty of each of them measured in terms of standard deviation. Some of the applied methods are known as classical in the fractal context (box-counting, rescaling-range and wavelets analyses, etc.) while the others have been recently developed by our Group. The combination of these techniques, coming from Fractal Geometry, Metrology, Informatics, Probability Theory and Statistics is termed in this paper Fractal Metrology (FM). We show the usefulness of FM for complex systems analysis through a case study of the soil's physical and chemical degradation applying the selected toolbox to describe and compare the structural attributes of three porous media with contrasting structure but similar clay mineralogy dominated by montmorillonites.
\end{abstract}

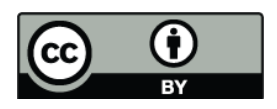

Correspondence to: $\mathrm{K}$. Oleschko (olechko@servidor.unam.mx)

\section{Introduction}

"If you cannot measure it you cannot manage it" (Cox, 2002). If you cannot measure something with known exactness and precision you cannot make unbiased decisions. The science of measurements is called Metrology and it deals with the theoretical and practical aspects of measurements (ISO, 2004; JCGM, 2008). The main goal for Metrology is to outline standardized ways in which natural constants and variable quantities can be measured to required accuracies (NIST, 2001). To date, some nine well-defined disciplines have been developed from the original Metrology, each one focusing on specific objectives (Fig. 1), with several emerging areas in the development phase (such as Roughness Metrology, Villarubia, 2005). Uncertainty and bias are dealt with in Statistical Metrology (Willink, 2005; Cox et al., 2008), while the features which affect the reliability of the measurements of linear and angular quantities in industrial production are analyzed by Dimensional Metrology (Curtis and Farago, 2007). The structure of uncertainty is analyzed in a reference way by Working Group 1 of the Joint Committee for Guides in Metrology (JCGM/WG1). We suggest that the study of complex and deeply interconnected Biogeosystems, whose behavior is defined by a common principle of self-organizing criticality (Beiró et al., 2008), requires special measurands (quantities to be measured, ISO, 2004) and a corresponding toolbox of reference measurement techniques to quantify the systems' scale invariance (SI), universality (UNI), nonlinearity (NL), complexity (COM), criticality (CR) as well as the uncertainty of their measurements.

Published by Copernicus Publications on behalf of the European Geosciences Union. 


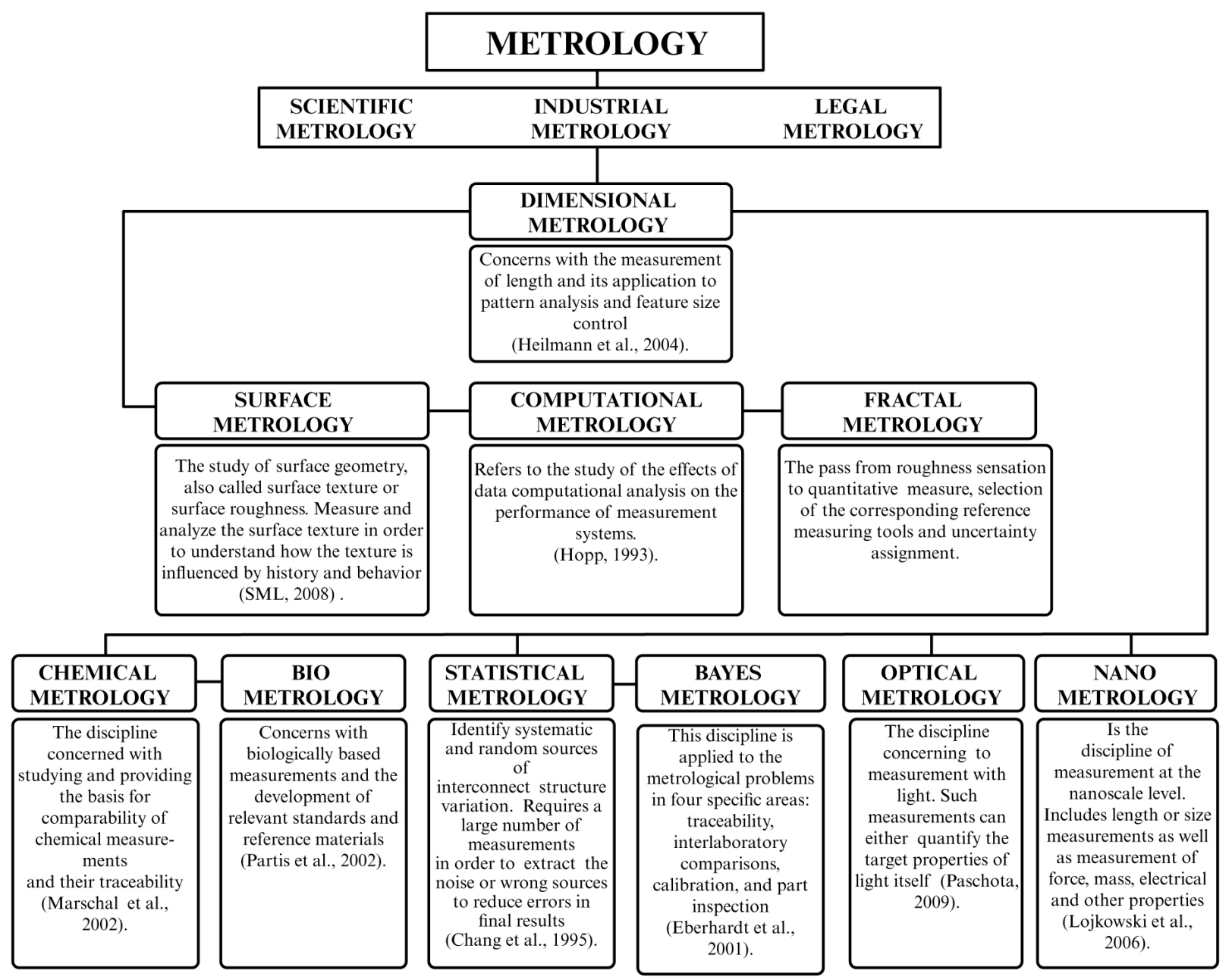

Fig. 1. Metrology division in sub-disciplines.

The lack of reference techniques, standards and quality control for the measurements of these basic attributes of complex systems, makes difficult any intergroup comparisons of the usually extensive data surveys, resulting in unsustainable decision-making. In this study, we combine some principles and techniques of Fractal Geometry, Metrology, Informatics, Probability Theory and Statistics to create a new branch of Metrology, what we propose to call Fractal Metrology, and introduce scale invariant roughness as the main measurand of SI, UNI, NL, COM and CR of complex systems (Oleschko et al., 2008).

The present study has three goals: (i) to propose (and check on an example) a step-by-step protocol for measuring the scale invariance of roughness on the structural patterns of a complex system (soil in our case) during the phase transition, paying special attention to the uncertainty of each used measurement technique; (ii) to compare statistically some new (designed by our group), as well as some common roughness measurement techniques on three soils with contrasting structural patterns, but with the same referencemineralogy; (iii) to document qualitatively (by visualization) and quantitatively (in terms of the Hurst exponent) the "symmetry breaking" (order/disorder transition) of soil aggregates under a degradation process (sodium salinization).

\section{Conceptual backgrounds}

\subsection{Complexity, criticality and roughness}

Under complexity, following Christensen and Moloney (2005), we understand the phenomenon when "the repeated application of simple rules in systems with many degrees of freedom gives rise to emergent behavior not encoded in the rules themselves". A well-known example for such repetitive rules is the random succession of faulting, uplift, subsidence and erosion leading to the wonderful variety of natural landscapes including those "that never were" (being the results of computer simulation, Mandelbrot, 1982; Korvin, 1992). 
The soil's aggregation is another example of this complexity when the combination of simple and known physical, chemical and biological rules, acting under different external and internal conditions, results in a diversity of pore/solid structural patterns whose geometry and topology are not derivable from these rules.

The term criticality refers to the behavior of the system at the point of phase transition, where no characteristic scale exists (and therefore there is pure scale invariance, Christensen and Moloney, 2005). In the case of soil, the aggregate's degradation occurs at a critical point and results in the solid/pore patterns' spatial re-arrangement.

Roughness is a basic common feature of all kinds of either real-world systems (natural, social, economical or technological) or mathematical objects. In the real-world, roughness characterization is mostly limited to visual judgment. The surface roughness appears as a set of apparently random peaks and valleys, resulting in the fine-texture irregularities due to the interaction of internal and external processes (El-Sonbaty et al., 2008). Smooth surfaces are rare in Nature (Majumdar and Bhushan, 1991), while rough ones have many useful properties ("rough skin is good" for suppressing air turbulence, see Monroe, 2006; Fransson et al., 2006). Roughness has a considerable effect on the contact of surfaces (Borri-Brunetto et al., 1999); it can influence $a d$ hesion (Wang et al., 2008), friction (Kim et al., 2006), wear (Bigerelle et al., 2007), and reflection (Verhoest et al., 2008). While surface roughness has a positive effect in increasing adhesion it is considered as an undesirable imperfection from the point of view of friction (Chandrasekaran and Sundararajan, 2004; Jensen, 2006). In each of these examples a small change in the distribution of heights, widths, or curvatures of the peaks has an important effect on the rough surface's behavior (Kim et al., 2006). Light scattering from optical coatings is the best example for how strongly processes could be affected by the roughness of interfaces (Germer, 2000). However, in spite of the great influence of surface roughness on system behavior its measurement is still a notable problem of Metrology (Villarubia, 2005; Van Gorp et al., 2007). Therefore, a quantitative measurement of surface roughness is essential for several applied and theoretical fields (Diehl and Holm, 2006), and would be especially useful in Biogeosciences. The question is: how to measure roughness in reference mode? We propose to extract the roughness from 2-D digital images, time series and signals by methods suitable for self-similarity and self-affinity analyses.

\subsection{Fractals and scale invariance}

No formal definition of fractals exists. Informally, Mandelbrot (2002) defines the fractals as irregular shapes, in either mathematics or the real world, with the property that each small part of them is a reduced-size copy of the whole. Mandelbrot emphasizes that the use of words fracture and fractal derived from the same root (fractus) is not a mere ac- cident. First by Mandelbrot et al. (1984), and then by numerous follow-up studies, it has been shown that the fractal dimension $D$ (the main distinctive attribute of a fractal) is an invariant measure of the roughness of fractures in metals and rocks (Mandelbrot, 2002). Mandelbrot proposed to view Fractal Geometry as a scientific approach to describe the sensation of rough versus smooth, as a "study of scale invariant roughness". Fractal Metrology has the same goal and measurand but it focuses on the selection and calibration of reference measurement techniques and their comparison in terms of uncertainty, as well as looking for the best-fitting measurement model.

Spatio-temporal invariance which is a main feature of natural nets and the basic concept of Physics is especially suitable to describe the structural patterns of complex systems. Recently, scale invariance as well has been found useful in applied sciences and for theoretical purposes.

We propose the scale invariant roughness as the main measurand of Fractal Metrology. Mandelbrot (2002, p. 5-6) argued that "much in nature is ruled by what used to be called pathology" but, fortunately, the latter "is not unmanageable". He continued: "This is so because it obeys a form of invariance or symmetry that overlaps Nature and Mathematics, and is called scale invariance or scaling that is central to my life work... The challenge is to explain why so many rough facets of Nature are scale-invariant". At this step, we define the main goal of Fractal Metrology as the pass from roughness sensation to quantitative measure (in agreement with Mandelbrot, 2002) by introducing the metrological fractal measurands, selection of the corresponding reference measuring tools and assignment of a realistic uncertainty to the measurements (compared by Student-t and Pearson's $r$ correlation analyses). This way, we shall accomplish the three main tasks of Metrology (NIST, 2001).

\section{Metrology}

Metrology is the science looking for the specific theoretical and practical aspects of the measurement and traceability, uncertainty and calibration carried out in the numerous applied and theoretical fields (JCGM, 2008). It was born to make comparisons based on quantitative measurements and directed to understand, interpret and make correct decisions about the system of interest. The selection of measurand is the first step in each measuring process. When this selection is made keeping in mind the strict standards of Metrology, the objects or system measurements become statistically precise and close to the true values of parameters.

\subsection{Fractal Metrology}

We designed Fractal Metrology to measure the degree of complexity and criticality of complex biogeosystems in terms of the roughness (main measurand) of their structural 


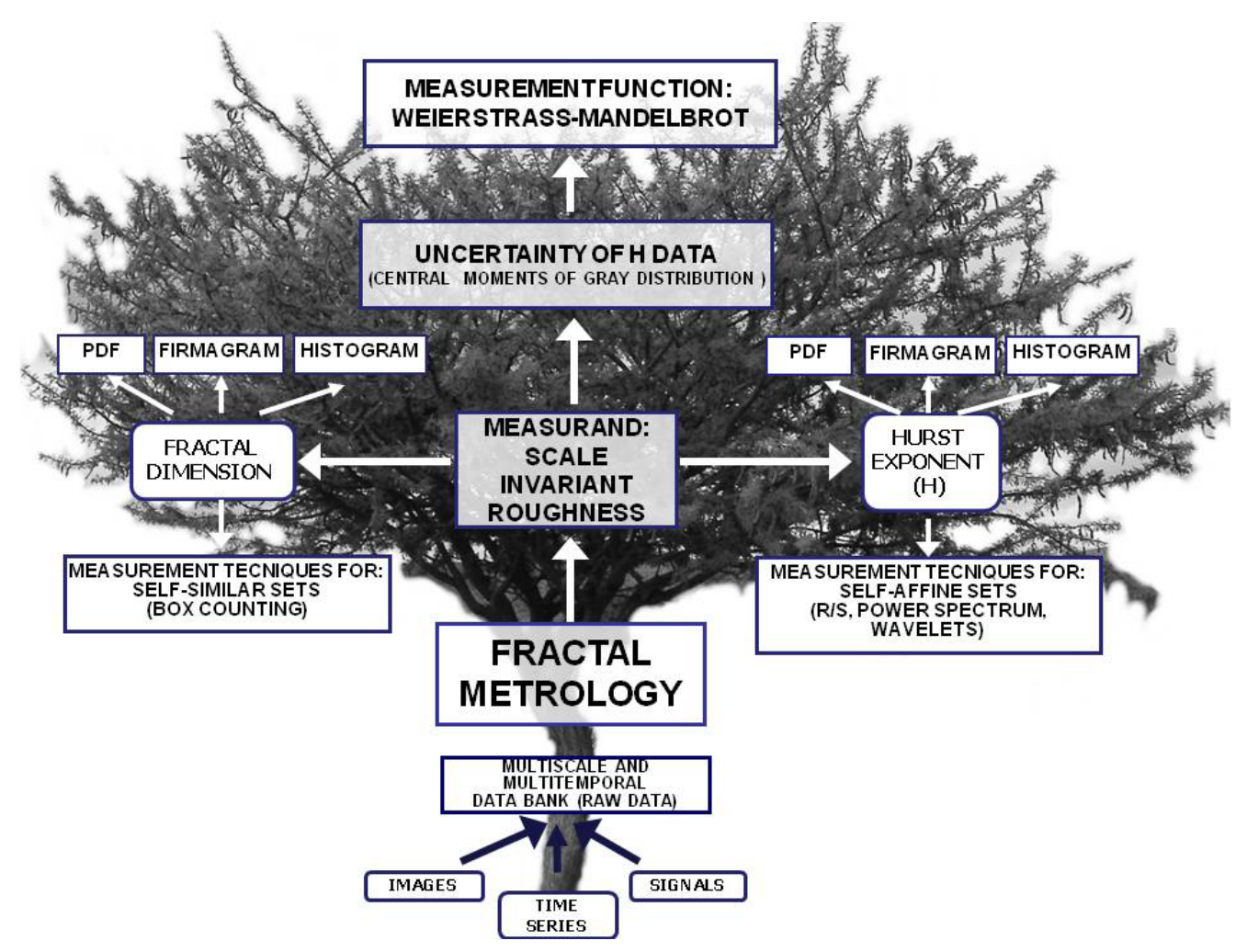

Fig. 2. Hierarchically organized Fractal Metrology construction.

patterns. Complex systems exhibit scaling properties which obey power laws (Katz, 2006; Plowman et al., 2007). In spite of the above-mentioned diversity of the branches of Metrology (Fig. 1), two main features of complex systems scale invariance and spontaneous symmetry breaking (Brink, 2008) - are still not measurable by standardized quantities (measurands) and reference measurement techniques.

The metrological description of each phenomena of interest comprises certain clearly defined steps (JCGM, 2008). The present research focuses on three of them: (1) the selection of the main measurand; (2) the comparison in terms of uncertainty between the known techniques for measurand quantification; (3) the selection of measurement model for measurements representation. These steps are visualized in Fig. 2. The image of a tree (Mezquite from Queretaro State, Mexico) was used to represent the branching structure (Dodds, 2010) of the information required by Fractal Metrology. This graph is suitable to design the step-by-step procedure for measurement of scale invariant roughness of multiscale and multitemporal images, time series or signals (input data). The distinctive feature of this information organization and management is a clear hierarchical and logical character of the system functioning. Three main roots are constituted by data banks which alimented the highly ramified tree constituted by known and new measurement techniques. All information is integrated by the unique trunk corresponding to the dimensionless measurands of roughness (fractal dimen- sions and corresponding Hurst exponents). These measurands can be extracted from the time series (measure space) or from the intensity's frequencies (probability space). The uncertainty is taken as the main indicator of efficiency of each compared technique to quantify the measurand with known precision. This way of analysis ensures optimal interaction among all elements of the net.

\subsubsection{Hurst exponent as the measurand of Fractal Metrology}

We selected the Hurst exponent as the main measurand of roughness and therefore of Fractal Metrology, because of its ability to express the asymptotic statistical properties of a random process $x(t)$ (Denisov, 1998), and because it merges local and global features of space/time anisotropy inside the unique variable called roughness. Proposed by the hydrologist Harold Edwin Hurst (1951), the classical rescaled adjusted range $R / S$-statistics has become a popular and robust technique for local and global dependence analysis (Mandelbrot, 2002). In time-series the Hurst exponent measures the growth of the standardized range of the partial sum of deviations of a data set from its mean (Ellis, 2007). Mandelbrot and Wallis (1968) have incorporated in the Hurst methodology ordinary least squares (OLS) regression techniques, and proposed to estimate the statistic over several subseries (windows) dividing the whole series length (Ellis, 2007). The 
Hurst exponent $(H)$ is related to the fractal dimension $(D)$ by a simple (conjectured) rule that first time appeared in Hardy's (1916) work:

$D=2-H$,

where 2 is the Euclidean dimension of the space where the fractal is embedded. The Hurst exponent is especially suitable to characterize stochastic processes (Mandelbrot and van Ness, 1968) from the point of view of scale invariance (Bassler et al., 2006). There are basic differences between persistent $(H>0.5)$ and antipersistent $(H<0.5)$ processes, while the white noise is characterized by $H=0.5$. Note that the $\mathrm{H}$ values tend to 0 when the roughness is growing.

\subsection{2 “Toolbox" of Fractal Metrology}

The soil roughness was extracted from digital images and measured on firmagram and probability density distributions by selected measurement techniques useful for self-affine set analyses.

\section{Optics of fractal objects: firmagram roughness}

Image digitization refers to the transformation of an apparently continuous image into discrete intensity values distributed at equally spaced locations across an xy-grid, called a raster (Pawley, 2006). The procedure results in an array of rows and columns which we (Oleschko et al., 2004) proposed to analyze as a one-dimensional array of data gathered inside the same column. Surface reflectance properties are among the most important attributes of matter. As a rough surface and its image have the same fractal dimension, the roughness can be statistically extracted from the images (Pentland, 1984; Gomez et al., 1998; Fleming et al., 2003; Puente, 2004; Korvin, 2005). We designed two methods to extract the digital image roughness, by converting the original image consisting of $N_{\mathrm{r}} \times N_{\mathrm{c}}$ pixels to a time series. Every pixel has a gray value $p_{i j}$ between 0 and 255. Here $i=1, \ldots, N_{\mathrm{r}}$ is the row-index, $j=$ $1, \ldots, N_{\mathrm{c}}$ is the column index, where $N_{\mathrm{r}}$ and $N_{\mathrm{c}}$ depend on the image size (mean image size in the present research was $1000 \times 874$ ). One way to convert an image to a time series is to rearrange all pixels row-wise into a 1-dimensional (1-D) array $F=\left\{p_{11}, p_{12}, \ldots, p_{1 N}, p_{21}, \ldots p_{2 N}, \ldots, p_{N 1}, \ldots p_{N N}\right\}$ of length $1000 \times 874$, what we call firmagram (Oleschko et al., 2004) and whose roughness can be measured by algorithms available for the analysis of self-affine sets. An other possibility is to consider the empirical histogram $n_{k}=\left\{\#\left(p_{i j}=k \mid k=0,1, \ldots, 255\right)\right\}$, or its normalized version, the empirical pdf $p_{k}=\left\{\frac{n_{k}}{N^{2}} \mid k=0,1, \ldots 255\right\}$. The series $p_{1}, p_{2}, \ldots, p_{255}$, extracted from each digital image of interest constitutes the time series for further fractal analysis. Note that, by definition, a stochastic process (or "random function") $\{x(t)\}_{\alpha}$ is a family of real- (or complex-) valued functions depending on a random parameter $\alpha$, where $t$ usually plays the role of time. In the case of image-analysis $t$ represents the pixel position (in case of firmagram analysis) or the intensity value (in case of $p d f$ analysis). The graphical representation of this column is the above-mentioned firmagram (Oleschko et al., 2004). The whole distribution of gray-tones (from 0- black, to 255- white) inside an image represents its global roughness (Fig. $4 \mathrm{Ab}, \mathrm{Bb}, \mathrm{Cb}$ ), while a baseline of each selected area refers to the local roughness. This dual representation visualizes how the image roughness is changing with scale, with an accuracy of one pixel. The Hist_Gen algorithm (Parrot, unpublished, 2003) scans the image from the first pixel on the top line until the final one on the bottom, building a column of the intensity values. The output files are in time series (.ts) format therefore the measurement of their roughness becomes a routine task (see Sect. 4).

\section{Histogram roughness}

The second way to extract the measurand for our research is using the histogram of gray scale values. The histogram is considered a precise way to summarize the statistical information associated with a complex system (Strauss, 2009; Tancrez et al., 2009). In the present work we used the algorithm Freq_Hist, written by Parrot (unpublished, 2003). The Freq_Hist output file consists of the frequencies of occurrence $(y)$ of each gray-intensity value $(x)$, forming a time series whose roughness can be measured by selected reference techniques. The results of the Hist_Gen are transferred to a Freq_Hist (.xls) file which contains the values of gray tones extracted from the original image, arranged in decreasing order and the respective probabilities to find a given gray value inside the image, constituting the effective probability density function $\left(\mathrm{PDF}_{\mathrm{ef}}\right.$ ) of the analyzed image (Fig. 4Ad, Bd, $\mathrm{Cd}$ ). The roughness of both PDFs is quantified as described in the Section on Measurement Techniques (see Sect. 4). The $\mathrm{PDF}_{\text {ef }}$ is compared with the modeled theoretical distribution by means of the @RISK (Palisade Corporation, 2005) software (Fig. 4Ae, Be, Ce).

\section{The roughness of the probability density function}

The Histogram is one of the most useful forms of summarizing random data for visual and statistical analysis ( $\mathrm{Lu}$ and Guan, 2009). It graphically represents data variability which is described in quantitative terms by the probability density function (Strauss, 2009). For a continuous function, the PDF expresses the probability that the variable of interest $X$ lies in an interval $(a b)$, (see, e.g. NIST/SEMANTECH, 2006):

$\int_{a}^{b} f(x) d x=\operatorname{Pr}[a \leq X \leq b]$. 
For a discrete distribution, the PDF represents the probability that the variable $X$ takes the value $x$. Note, that when displayed, the PDF's graph has the same appearance as the histogram (Fig. 4Ad, Bd, Cd, 4Ae, Be, Ce).

In our research to quantify the fractal behavior of PDF, in addition to the PDF estimation by means of Freq_Hist, the statistical analysis of the gray-tone distribution across each analyzed image has also been accomplished by the commercial software @ RISK 4.5 add-in for Microsoft Excel (Palisade Corporation, 2005). The tools of Risk Analysis have been used since long for the analysis of financial data, but rarely applied in Natural Sciences. We have found @RISK a user-friendly software (except its rather high price!) suitable for Biogeoscience studies because of its precision and relative simplicity.

The @RISK 4.5 package selects the best fit to the experimental data function from among 37 different theoretical probability distributions (Normal, Lognormal, Logistic, Beta, Gamma, Pareto, etc.). The algorithm is based on a Monte-Carlo simulation technique which replaces the uncertain or unknown values of an experimental dataset by a range of more probable values. The list of five selected, best fitting functions are displayed automatically, the first one being the most probable for the studied data. To create a histogram, the software finds the maximum and minimum values of a data range, divides the range into classes whose level of importance depends on the probability of occurrence of values, defined as $\{p\}=\left\{p_{1}, p_{2}, \ldots, p_{n}\right\}=$ data rank array. In @RISK 4.5 (Palisade Corporation, 2005) the probability density function is used to construct the frequency distribution from an infinitely large set of values where the class size is becoming infinitesimally small. The visual similarity between PDFs constructed by Freq_Hist and @RISK can be observed in Figs. 4Ad, Bd, Cd and 4Ae, Be, Ce, except the roughness which is always higher in the former case.

\section{Measurement techniques of Fractal Metrology}

There is a legion of fractal descriptors suitable to quantify the specific attributes of complex systems (Oleschko et al., 2004). For instance, the fractal dimension $(D)$ measures the set's space-filling ability (Mandelbrot, 1982); the degree of its translation invariance is quantified by lacunarity $\Lambda$ (Pendleton et al., 2005; Feagin, 2003; Feagin et al., 2007); the continuity and tortuosity of the pore and solid networks are measured by random-walk fractal dimensions (Korvin, 1992; Rodriguez-Iturbe and Rinaldo, 1997), or spectral dimension or fracton (Orbach, 1986). The main advantages and problems of fractal descriptor measurements have been described in details in some by now standard (Korvin, 1992; Barton and La Pointe, 1995; Falconer, 1997; Turcotte, 1997, etc.) as well as recent (Tél and Gruiz, 2006) books. There are several useful reviews comparing the algorithmic aspects of these measurements and the performance of each fractal
Table 1. Mean Hurst exponents $(H)$, fractal dimensions $(D)$ and standard deviations $(S)$ measured by box counting method for seventeen analyzed images of three compared soils and their respective firmagrams.

\begin{tabular}{lllllll}
\hline Image & \multicolumn{3}{c}{$D_{\text {box }}$ (mean values) } & \multicolumn{3}{c}{$D_{\text {box }}$ firmagram (mean values) } \\
& $H$ & $D$ & $S$ & $H$ & $D$ & $S$ \\
\hline Chernozem & 0.083 & 1.917 & 0.0165 & 0.1525 & 1.8475 & 0.0015 \\
Solonetz & 0.0935 & 1.9065 & 0.0145 & 0.127 & 1.873 & 0.008 \\
Clay & 0.125 & 1.875 & 0.016 & 0.1005 & 1.8995 & 0.0025 \\
\hline
\end{tabular}

dimension: for instance, the boundary fractal dimension is treated in Klinkenberg (1994) and Gallant et al. (1994); selfaffine time series analysis in Malamud and Turcotte (1999) and Pelletier and Turcotte (1999), while the correlation dimension was the subject of Kogan's (2007) detailed study. A comparison of computer-simulated examples was given by Behery (2006). The compilation of Sun et al. (2006, Table 1), focusing on the techniques used for the fractal dimension analyses of the surface features extracted by remote sensing, is especially useful for summarizing and comparing the different techniques.

Mandelbrot (2002) proposed to put the most important fractal analysis techniques into a "toolbox", just as the tools of the electricians and plumbers. The "power-law" figuring in the probability distribution $\operatorname{Pr}\{U>u\} \sim u^{-\alpha}$ describing the distribution of a system's attributes having a size $U>u$ can be used in Fractal Metrology as a superior tool for fractal modeling. Levitz (2007) used the notion "basic toolbox" to capture forms and patterns, while we applied the term "toolkit" as more proper for applied sciences (in Oleschko et al., 2010). For Fractal Metrology we propose to use the original term "toolbox" (or effective toolbox), to honor the pioneering works of Mandelbrot (2002). We shall put inside this box some tools designed by us in addition to the common fractal techniques of one of the available commercial software - Benoit (1.3) (Trusoft, 1999, one of its early versions was reviewed by Seffens, 1999). Each Benoit technique is based on some specific relationship (such as: power law) established theoretically, empirically, or by computational experiment between a system attribute and the scale of its observation. The box-counting ( $\left.D_{\mathrm{box}}\right)$, perimeter-area $\left(D_{\mathrm{p}}\right)$, information $\left(D_{\mathrm{i}}\right)$, mass fractal $\left(D_{\mathrm{m}}\right)$, and ruler $\left(D_{\mathrm{r}}\right)$ dimensions (and corresponding Hurst exponents) are designed for self-similar sets or curves, while the rescaled range $\left(D_{\mathrm{RS}}\right)$, power spectrum $\left(D_{\mathrm{PS}}\right)$, roughness/length $\left(D_{\mathrm{r}}\right)$, variogram $\left(D_{\mathrm{v}}\right)$, and wavelet $\left(D_{\mathrm{w}}\right)$ dimensions are used for self-affine traces or time series (Trusoft, 1999). The following discussion will involve only four of the mentioned techniques. 

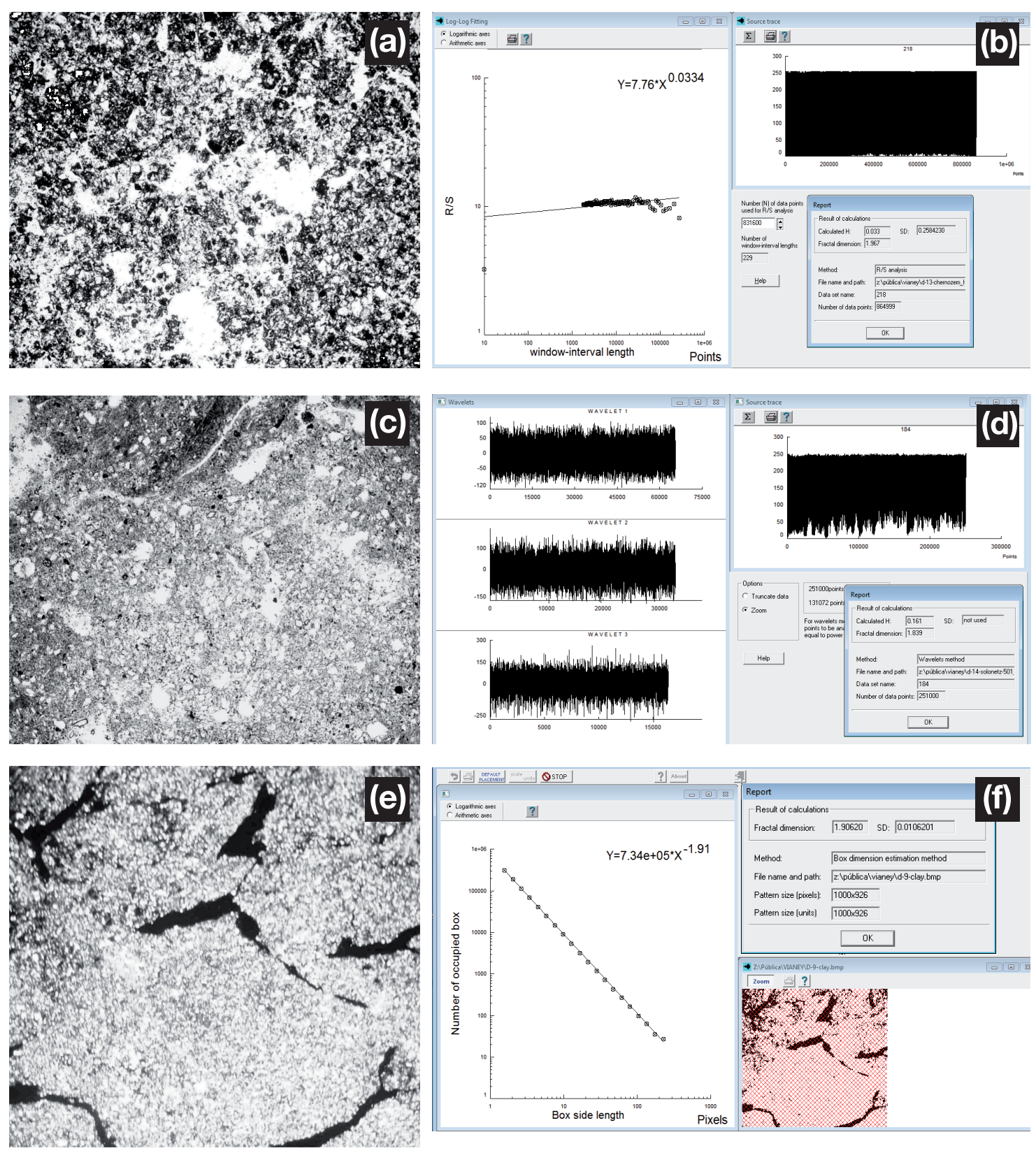

Fig. 3. Benoit' (Version 1.3) outputs of the compared methods: $R / S$ analysis (b), wavelets (d) and box counting (f) applied to images of the Chernozem (a), Solonetz (c) and Clay (e) visualizing the details of each procedure.

\subsection{Box dimension $\left(D_{\text {box }}\right)$}

The size of a self-similar fractal set displays a power-law relationship with the measurement scale where the fractal dimension is the exponent of the power-law (Tang and Marangoni, 2006). The Box Dimension technique is the classical way to prove the fractal behavior of the studied mathematical, computer-simulated or real physical set and is used in this work to measure the roughness from the space-filling ability of solid and pore networks. In this technique, the counting of boxes containing pixels of the object is accomplished, considering the box as occupied if at least one analyzed intensity value belongs to the box. The following equation is basic for $D_{\text {box }}$ calculation:

$$
N(d) \approx \frac{1}{d^{D_{\mathrm{box}}}},
$$

where $N$ (counted for a set of box sizes with different orientation) is the number of those boxes of linear size $d$ which contain at least one point of the structure (Fig. 3f). 


\subsection{Rescaled range analysis $\left(D_{R / S}\right)$}

The $R / S$ analysis is one way to characterize the selfsimilarity properties of time series through the Hurst exponent (Scipioni et al., 2008). This traditional method can be described in terms of the range of partial sums of deviations of values from the mean of a time series, normalized by its standard deviation (Alvarez-Ramirez et al., 2008). The Rescaled-Range $R / S(w)$ is defined as (TruSoft, 1999):

$R / S(w)=\left\langle\frac{R(w)}{S(w)}\right\rangle$,

where $w$ is the window length; $R(w)$ is the range of values inside the sampled interval; $S(w)$ is the average standard deviation, angular brackets denote expected values. The following equation shows the power-law relation which can be established between the $R / S$ ratio and window length via the Hurst exponent $H$ :

$R / S(w) \propto w^{H}$.

The linearity of the double logarithmic plot of $R / S(w)$ as a function of $w$ reveals a scaling law, where $H$ is the Hurst exponent which is obtained from the slope of the straight line. The relationship between fractal dimension and Hurst exponent is given by Hardy's (1916) conjecture mentioned in Sect. 3.1.1.

\subsection{Power spectrum $\left(D_{\mathrm{PS}}\right)$}

A powerful method to extract hidden structural information (such as: periodicities and persistence) from a fluctuating time-series is to calculate its power spectrum ( $\mathrm{Su}$ and $\mathrm{Wu}$, 2007). The power spectrum method gives a scale invariant measure of fractal dimension since the log-log slope of the high-frequency range of the power spectrum is invariant to arbitrary rescaling of the input (Wilson, 1997). Usually, the Fast Fourier Transform (FFT) is applied to estimate the power spectrum (Dimri and Prakash, 2001). To obtain an estimate of the fractal dimension, the power spectrum $P(k)$ (where $k=2 \pi / \lambda$ is the wavenumber, and $\lambda$ is the wavelength) is first calculated and plotted on a double logarithmic plot as $P(k)$ versus $k$. If the time-series is self-affine, this plot should follow a straight line for large wavenumbers, with a negative slope $-b$ which is estimated by regression. The exponent $-b$ is related to the fractal dimension $D_{\mathrm{PS}}$ as (TruSoft, 1999):

$D_{\mathrm{PS}}=\frac{5-b}{2}$.

\subsection{Wavelets $\left(D_{\mathrm{W}}\right)$}

Wavelets are localized functions of mean zero, constructed by the linear combination of scaling functions (Bakucz and Krüger-Sehm, 2009). They are especially useful for compressing images where they are in some ways superior to the conventional Fourier transform (Weisstein, 2010). The wavelet transform of a self-affine trace is also self-affine (Rehman and Siddiqi, 2009). The characteristic measure of wavelet variance analysis is the wavelet exponent, $H_{\mathrm{w}}$ (Malamud and Turcotte, 1999). Wavelets are implemented using trigonometric functions that are oscillating around zero in a non-smooth sweep, and localizing them in the frequency space (Jones and Jelinek, 2001). Consider $n$ wavelet transforms all of them with a different scaling coefficient $a_{i}$, let $S_{1}, S_{2}, \ldots S_{n}$ be their standard deviations from zero. Define the ratios $G_{1}, G_{2}, \ldots, G_{n-1}$ of the standard deviations as $G_{1}=S_{1} / S_{2}, G_{2}=S_{2} / S_{3}, \ldots, G_{n-1}=S_{n-1} / S_{n}$, and compute the average value of $G_{i}$ as (TruSoft, 1999):

$G_{\mathrm{avg}}=\frac{\sum_{i=1}^{n-1} G_{i}}{n-1}$.

The Hurst exponent $(H)$ is $H=f\left(G_{\text {avg }}\right)$, where $f$ is a heuristic function which describes $H$ by $G_{\text {avg }}$ for stochastic self-affine traces (TruSoft, 1999). The mother wavelet in Benoit 1.3 is a step function. Malamud and Turcotte (1999) underlined that wavelet analysis does not share the inherent problems of power spectrum analysis, such as windowing, detrending etc.

\section{Measurement uncertainty}

We propose to measure uncertainty in terms of the standard deviation $(\delta)$. The $H$ and $\delta$ values extracted by selected Benoit techniques from the original digital images, firmagrams and PDF were subjected to Pearson's $r$ and Student's-t statistical analyses in order to estimate the statistical significance of the differences between them.

Comparative analysis of the four selected reference measurement tools is realized in the present research following the Guide to the Expression of Uncertainty in Measurement (GUM), published by the Joint Committee for Guides in Metrology (JCGM, 2008). The term "measurement uncertainty" is used in its broadest sense as a doubt defining it as a parameter, associated with the result of a measurement, that characterizes the dispersion of the values that could be reasonably attributed to the measurand (JCGM, 2008, p. 2). The GUM recognizes two types of measurement errors (systematic and random) putting them on a probabilistic basis through the concept of measurement uncertainty (JCGM, 2009). The latter is described as the measure of how well one believes one knows the measurand value (JCGM, 2009, p. 3).

We propose to measure the uncertainty of fractal analyses in terms of the standard deviation $(\delta)$ of the Benoit results.

The statistical significance of the differences in uncertainties of the Benoit's data was quantified by Student-t and Pearson's $r$ correlation analyses. The Pearson's $r$ correlation 
matrix (computed by the MINITAB Software, 1998) was constructed as:

$r_{X Y}=\frac{\sum_{i=1}^{n}\left(X_{i}-\bar{X}\right)\left(Y_{i}-\bar{Y}\right)}{(n-1) S_{X} S_{Y}}$,

where $X, Y$ are all possible pairs of the compared variables, see Tables 3, 6, 7. Here, $\bar{X}$ and $\bar{Y}$ are mean values, $S_{X}$ and $S_{Y}$ are standard deviations. Student's-t-test was carried out for paired variables in the SPSS Inc. (2004) environment.

For the @ RISK results we carried out the statistical comparison of the significance of the obtained differences in a few statistics built from the first four moments: mean, variance, kurtosis and skewness.

The standard deviations of all used techniques (except the wavelets) were analyzed by the same statistical tests but independently of $H$. Additionally, the Pearson's $r$ and Student'st-tests were applied to the three compared soils of contrasting genesis, looking for a correlation between the roughness of their images. Finally, the same two statistical tests were applied to the four statistics yielded by @ Risk PDF analysis.

\section{Results and discussion}

In the present research the collapse of the solid/pore structural pattern of Chernozem was studied under a common agricultural degradation process, salinization, leading to an emergent new unfertile soil, namely Solonetz. The structural patterns of both soils are compared with the pure "Chocolate" Clay (a kind of clayey deposit in Russia with chocolate color) composed by minerals of the montmorillonite group. The main difference between these three soils is the origin of the dominating cation inside the CEC (Cation Exchange Complex): calcium in the Calcic Chernozem, and sodium in Solonetz and Chocolate Clay. The Solonetz was formed from the Chernozem inside the same Chernozem-Solonetz pedological complex (Oleschko, 1981), while the Chocolate Clay was taken as the example of a reference-matrix which has never been involved in the aggregation process but has the similar texture and clay mineralogical composition (Vadyunina et al., 1980). We are looking for critical behavior in the Chernozem-Solonetz complex, comparing the soil structural patterns before and after the transition from the totally aggregated (State I, Chernozem) to massive (State II, Solonetz) structure (Oleschko, 1981). During this transition, the structural pattern of Chernozem, a highly fertile soil with perfectly permeable sponge structure (Fig. 3a), gets transformed into the massive structure (Fig. 3c) of the unfertile bad land (Solonetz) with similar to Chocolate Clay appearance. We focused our attention on those structural attributes of Chernozem which have remained unchanged during the transition to Solonetz, and tested the ability of Fractal Metrology to measure the differences and similarities between the compared soils.

\subsection{Experimental setup}

Seventeen micromorphological images of three soils with contrasting structural patterns but similar clay content and mineralogy (Oleschko, 1981) were used for the statistical comparison of the measurement techniques selected for inclusion in Fractal Metrology. The Chernozem-Solonetz pedological complex was sampled in the same agricultural field (Tambov State, Russia). The undisturbed samples $(8 \mathrm{~cm} \times 4 \mathrm{~cm})$ were collected with specially designed samplers from the arable horizon of each studied soil. All samples were taken at field moisture in order to conserve the soil's structure. In the laboratory, samples were dried by the acetone replacement method. Thin sections $(2 \mathrm{~cm} \times 4 \mathrm{~cm})$ with $30 \mu \mathrm{m}$ thickness, were prepared by the (then standard in the Soviet Union) petrographic procedure (Parfenova and Yarilova, 1977; Brewer, 1964) from the samples sectioned horizontally making sure that the natural solid-pore distribution anisotropy derived from the tillage practices is preserved (Fig. 3a, c, e). The thin sections were analyzed under petrographic (Carl Zeiss) microscope, taken all digital images under magnification 10× (Oleschko, 1981).

The Chernozem and Solonetz are located inside the patches of a typical mosaic of a man-induced Bad Lands landscape. However, the Chernozem is the black soil with the highest known level of sponge-type structure development (Phase 1), while the Solonetz is a saline-sodium soil with typical massive pattern and ephemeral fractures derived from the alternating wetting (expansion) and drying (contraction) processes (Phase 2). Solonetz had originated from the Chernozem as a result of chemical degradation due to unsustainable irrigation with saline water. Therefore, the experimental setup was focused to capture the critical behavior and phase transition of the soil's structural pattern during this degradation, applying the above-described Fractal Metrology techniques and preserving the original, representative structural patterns' anisotropy.

Figures $3 \mathrm{a}, \mathrm{c}$ and $4 \mathrm{Aa}, \mathrm{Ba}$ show the representative examples of micromorphological images of Chernozem (Figs. 3a and 4Aa) and Solonetz (Figs. 3c and 4Ba), visualizing their contrasting structural patterns that have resulted in statistically different physical properties (Table 2) and soil behavior. The loss of the original quality is related to the structure's collapse in response to the drastic changes which occurred inside the Cation Exchange Complex where the calcium, dominating in Chernozem, had been replaced by sodium, resulting in Solonetz formation. The phase transition from the highly connected to massive pattern with water-unstable structure and ephemeral fractures has occurred when the sodium concentration exceeded the permissible (critical) level resulting in the catastrophic decrease of the macro- and microaggregates' stability. Because of the universality of phase transitions (Stanley, 1971), we expected similar Hurst exponents in case of both soils regardless of some local details. In our case the divergences of the order 

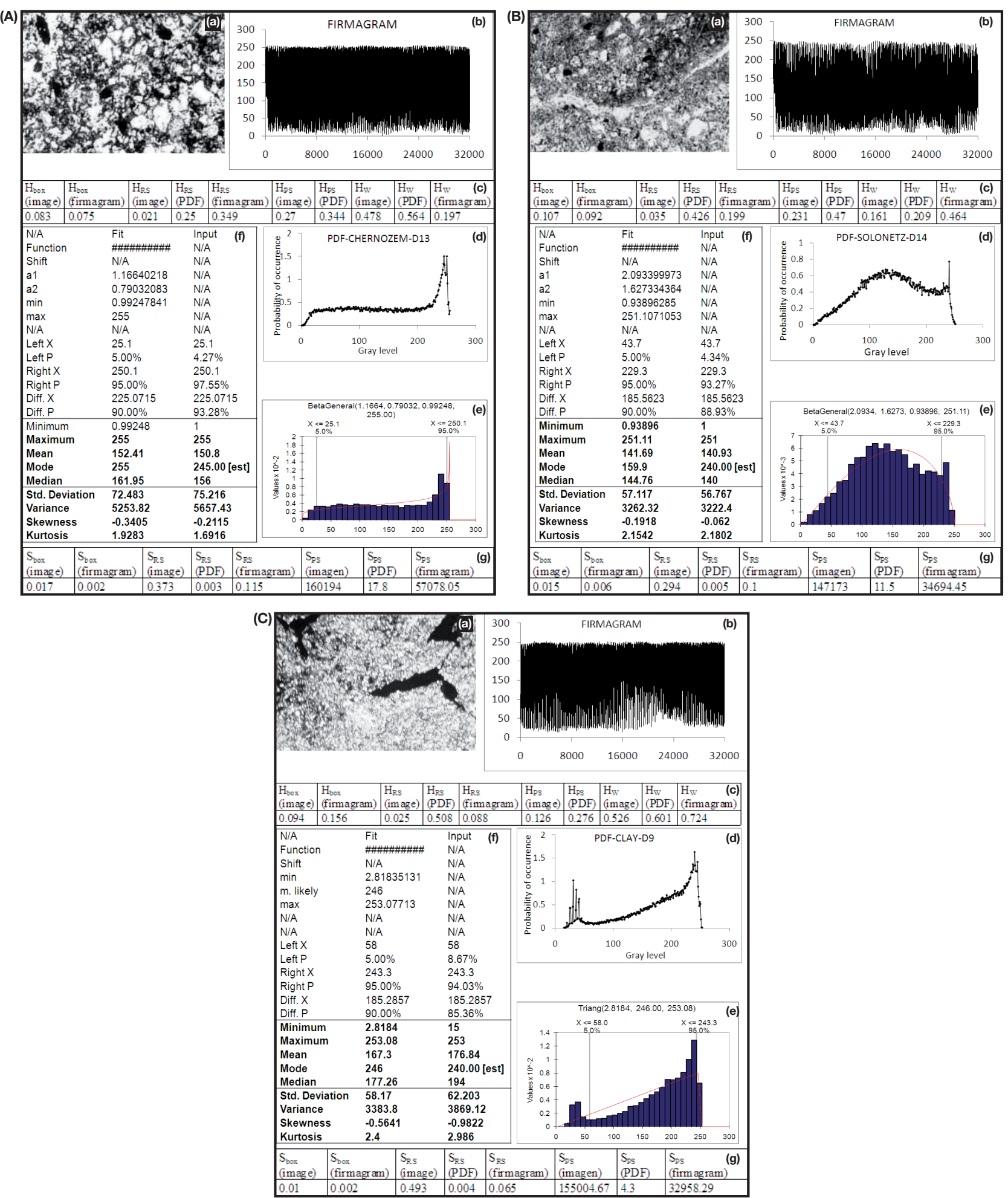

Fig. 4. Firmagrams (Ab, Bb, Cb) extracted from the micromorphological images of three studied soils: Chernozem (Aa); Solonetz (Ba) and Clay $(\mathbf{C a})$, with contrasting structural patterns. The roughness values expressed in terms of Hurst $(H)$ exponent $(\mathbf{A c}, \mathbf{B c}, \mathbf{C c})$ and their standard deviation (Ag, Bg, $\mathbf{C g}$ ) for the compared techniques. The distributions of gray intensities (Ad, Bd, Cd) are identified as visual singularities of the image: PDF (Ad, Bd, Cd). These differences are detectable by eye when the graphs of data are fitted to the most probable theoretical distribution by software @ Risk (Ae, Be, Ce), and with the central moments calculated by the same software (Af, Bf, Cf). 
Table 2. The microaggrgate composition of the Chernozem and Solonetz soils at different depths.

\begin{tabular}{llrrrrrrr}
\hline & & \multicolumn{6}{c}{ Microaggregate fraction $(\%)$} \\
\cline { 3 - 8 } Soil & Depth $(\mathrm{cm})$ & $1-0.25$ & $0.05-0.01$ & $0.01-0.005$ & $0.005-0.001$ & $<0.001$ & $<\mathbf{0 . 0 1}$ & $>\mathbf{0 . 0 1}$ \\
\hline \multirow{2}{*}{ Chernozem } & $(0-20)$ & 0.0 & 46.9 & 17.8 & 8.2 & 2.5 & $\mathbf{2 8 . 5}$ & $\mathbf{7 1 . 5}$ \\
& $(40-50)$ & 0.2 & 44.8 & 15.8 & 17.7 & 2.7 & $\mathbf{3 6 . 2}$ & $\mathbf{6 3 . 8}$ \\
\multirow{3}{*}{ Solonetz } & $(0-20)$ & 0.0 & 46.5 & 21.5 & 13.5 & 9.9 & $\mathbf{4 4 . 9}$ & $\mathbf{5 5 . 1}$ \\
& $(40-50)$ & 0.0 & 21.1 & 17.6 & 12.3 & 45 & $\mathbf{7 4 . 9}$ & $\mathbf{2 5 . 1}$ \\
\hline
\end{tabular}

parameters at the critical $\mathrm{Na}$ content $C_{\mathrm{Na} \text {,crit }}$ scale near the critical point as $\sim\left|C_{\mathrm{Na} \text {, crit }}-C_{\mathrm{Na}}\right|^{-\lambda}$. We tried to capture and visualize this trend for the compared soils, measuring it by selected techniques from the Fractal Metrology toolbox. The final comparison was carried out between the structural patterns of both soils and the Chocolate Clay whose massive microstructure had never passed through an aggregation process and whose mineralogy is similar to the studied soils parent materials (Figs. 3e and $4 \mathrm{Ca}$ ).

\subsection{Structural patterns comparison by fractal measurands}

The mean values of the box fractal dimensions for the compared soils, extracted from the original images and the negative images of the firmagram (Benoit's box counting algorithm is working only on the white part of an image, Fig. 3f) were statistically similar and close to the value of 1.89 , the fractal dimension of the Sierpinski carpet (Korvin, 1992). $D_{\text {box }}$ varies between 1.8475 (Chernozem's firmagram) and 1.917 (digital image of Chernozem, Table 1). The former value is the lowest and the latter is the highest among the compared soil samples. These trends coincide with what we intuitively predicted: the more aggregated soil has the highest roughness on macro scale (level of structural fractals) but lowest density of solids on micro scale (level of textural fractals, where the Chocolate Clay has the most densely distributed pattern of solid particles possible in the Euclidean space). The division of fractals into structural and textural was accomplished by Avnir and Farin (1984), using their roughness as indicator for the particles' spatial arrangement. The surface of the fine particles has the same smoothness in all compared soils which build the observed complex pore/solid networks (Dathe et al., 2001).

The highest difference in box fractal dimensions and corresponding Hurst exponent (and therefore in roughness) was documented for Chernozem and Chocolate Clay where these fractal measurands, extracted from the digital images, have differences of 0.042. Note that this small difference in dimension can be translated into a significant porosity change.
Table 3. Pearson-r correlation matrix for the Hurst exponent of three studied soils.

\begin{tabular}{llll}
\hline & Clay & Solonetz & Chernozem \\
\hline Clay & 1 & $0.872^{*}$ & $0.909^{*}$ \\
& & 0.001 & 0 \\
Solonetz & 1 & $0.870^{*}$ \\
& & & 0.001 \\
Chernozem & & 1 \\
\hline
\end{tabular}

* Correlation is significant at the 0.01 level (2-tailed).

The observed data variation is low, with highest mean standard deviation of 0.0165 obtained for $D_{\text {box }}$ of Chernozem (original image) and the minimal $(0.0015)$ for the negative of the firmagram. The discussed roughness differences between compared soils were not significant statistically (Tables 3 and 4). We concluded that from the statistical point of view all compared porous materials should be defined as similar in the space filling ability of their solid and pore patterns, and in their roughness calculated by the reference box fractal dimension. Note that the box counting analysis of the firmagram extracts more precise information about the matrix density (mutual distribution of solids and pores) inside the original images.

The apparent independence of the box counting dimension on soil genesis for the studied pedological complex compared with Chocolate Clay (a porous material, strictly speaking not a soil) can be interpreted as empirical evidence for some generic features (universality?) of the roughness of these materials with similar clay mineralogy (micro-scale) but contrasting appearance of soil structural patterns and drastically different physical behavior on macro-scale. In this case it is possible to speak about the universal critical exponents of soil aggregation process which stay constant during the phase transition from the aggregated (Chernozem) to the dispersed (Solonetz) state.

Therefore, neither the box fractal dimension nor its standard deviation was able to detect any statistically significant differences in the roughness of digital images and 
Table 4. Student-t-test for the Hurst exponents of three compared soils.

\begin{tabular}{|c|c|c|c|c|c|c|c|c|}
\hline & \multicolumn{8}{|c|}{ Paired Differences } \\
\hline & \multirow[t]{2}{*}{ Mean } & \multirow[t]{2}{*}{$S$} & \multirow[t]{2}{*}{ SEM } & \multicolumn{2}{|c|}{$\begin{array}{l}95 \% \text { Confidence Interval } \\
\text { of the Difference }\end{array}$} & \multirow[t]{2}{*}{$t$} & \multirow[t]{2}{*}{ Df } & \multirow[t]{2}{*}{ Sig. (2-tailed) } \\
\hline & & & & Lower & Upper & & & \\
\hline Clay-Solonetz & 0.025 & 0.101 & 0.032 & -0.047 & 0.097 & 0.786 & 9 & 0.452 \\
\hline Clay-Chernozem & 0.021 & 0.086 & 0.027 & -0.041 & 0.083 & 0.771 & 9 & 0.461 \\
\hline Solonetz-Chernozem & -0.004 & 0.087 & 0.028 & -0.066 & 0.058 & -0.146 & 9 & 0.887 \\
\hline
\end{tabular}

firmagram of the compared soils. We speculate that these results indicate the need to include further fractal measurands (most importantly: some agreed-upon standard measure of lacunarity) into our proposed toolbox.

The third technique used in the present study, namely the PDF roughness measurement, was not statistically sensitive to soil structural dynamics. In spite of the clear visual differences detectable on the PDF morphology of the three compared soils, the statistics derived from their four central moments (mean, standard deviation, skewness and kurtosis) were not statistically different. It should be mentioned that PDF is invariant to the interchange of any two pixels inside the image. Note that the firmagram and the power spectrum are also more sensitive to local short-wavelength properties than to the larger structural features. Therefore, we concluded that the macropores (with a diameter of $\geq 1 \mathrm{~mm}$ ) are not displayed in statistically representative way in a single digital image. Dathe et al. (2001) came to the similar conclusion. Our previous physical experiments, and corresponding computer simulations, have shown similar trends for soils of different genesis (Oleschko et al., 2000), resulting in similar values of the corresponding fractal parameters (Oleschko et al., 2002, 2003).

In Table 2 the microaggregate compositions of the Chernozem and Solonetz estimated by the reference pipette method are compared for two genetic horizons. The high content of physical clay (the physically active fraction of particles with size $<0.01 \mathrm{~mm}$, considered as most important for microaggregation) in both soils ensures their suitability to form clusters of fine particles (microaggregates). Notwithstanding, the differences in the nature of the dominant cation in CEC are responsible for the contrasting physical and chemical properties of Chernozem and Solonetz. We speculate that the similarity in roughness between these soils measured by box-counting technique is due to their high physical clay content, which increased significantly with depth (Oleshko et al., 1980), while the effect of pore macrofeatures on the roughness measured pixel by pixel, and therefore on Hurst exponent, is masked by the high microporosity.

The Pearson's $r$ analysis as well as the Student-t-test confirmed the strongly significant correlations between the roughness of all compared soils and therefore non-significant differences in $H$, independently of the applied measurement techniques (Tables 3 and 4).

\subsection{Uncertainty of fractal measurement techniques}

As the second step of metrology measurement protocol, the Hurst exponent values, extracted from the images, firmagrams and PDFs by Box Counting, Rescaled-Range, Power Spectrum and Wavelets techniques were compared as regards to the mean value of the Hurst exponent and its standard deviation (Table 5). The $H_{\mathrm{RS}}$ extracted from the original images transformed into time series has higher mean value in Chernozem (0.064) than in Solonetz (0.039) and Clay (0.031). The mean standard deviation is also higher for the $H_{\mathrm{RS}}$ of Chernozem (0.742) than for Solonetz (0.362) and Chocolate Clay (0.377). We note that the Hurst exponent of the Chernozem has a standard deviation measured by Rescaled Range technique $(0.742)$ which is much larger than for box counting (0.017).

As in the case of box counting (Table 5), the standard deviation was smaller for $H_{\mathrm{RS}}$ extracted from the firmagrams, being equal to 0.193 for Chernozem and minimal for Clay (0.104). The mean value of $H_{\mathrm{RS}}$ measured for the firmagrams in three soils was 0.229 , and therefore the corresponding mean fractal dimension was $D_{\mathrm{RS}}=1.771$. The values of roughness measured by power spectrum method on the original images and firmagrams were comparable with those obtained by the $R / S$ technique (except the "Clay" samples where the fractal dimension has reached the topological limit of 2): the mean value of $H_{\mathrm{PS}}$ is equal to 0.208 for images. For all techniques the roughness information extracted from the PDF was noisier than in case of original digital images and firmagrams extracted from these. In spite of the listed differences between the differently measured Hurst exponents, these were not statistically significant only for two compared standard deviation pairs (Tables 5, 6). The $H$ values measured by Wavelets technique fluctuated around 0.5 (the Hurst exponent value of white noise) similarly to the above-discussed PDF case. We concluded that the Wavelets technique was not sufficiently precise for the roughness measurement of the digital images. 
Table 5. Hurst exponent $(H)$ and standard deviation $(S)$ for each soil type and measurement technique used.

\begin{tabular}{lllll}
\hline & Total mean & Chernozem & Solonetz & Clay \\
\hline$H_{\text {box (image) }} / S_{\text {box (image) }}$ & $0.100 / 0.014$ & $0.083 / 0.017$ & $0.101 / 0.014$ & $0.117 / 0.013$ \\
$H_{\text {box (firmagram) }} / S_{\text {box (firmagram) }}$ & $0.125 / 0.004$ & $0.153 / 0.002$ & $0.119 / 0.006$ & $0.106 / 0.004$ \\
$H_{\mathrm{RS}(\text { image) }} / S_{\mathrm{RS}(\text { image) }}$ & $0.044 / 0.493$ & $0.064 / 0.742$ & $0.039 / 0.362$ & $0.031 / 0.377$ \\
$H_{\mathrm{RS}(\mathrm{PDF})} / S_{\mathrm{RS}(\mathrm{PDF})}$ & $0.444 / 0.007$ & $0.424 / 0.006$ & $0.505 / 0.010$ & $0.404 / 0.006$ \\
$H_{\mathrm{RS}(\text { firmagram) }} / S_{\mathrm{RS}(\text { firmagram) }}$ & $0.229 / 0.141$ & $0.270 / 0.193$ & $0.201 / 0.127$ & $0.217 / 0.104$ \\
$H_{\mathrm{PS}(\text { image }} / S_{\mathrm{PS}(\mathrm{image})}$ & $0.208 / 144302.145$ & $0.151 / 133766.650$ & $0.233 / 146878.879$ & $0.241 / 152260.906$ \\
$H_{\mathrm{PS}(\mathrm{PDF})} / S_{\mathrm{PS}(\mathrm{PDF})}$ & $0.417 / 11.244$ & $0.454 / 10.960$ & $0.435 / 14.941$ & $0.366 / 7.831$ \\
$H_{\mathrm{PS}(\text { firmagram) }} / S_{\mathrm{PS}(\text { firmagram) }}$ & $0.048 / 40068.195$ & $0.121 / 48708.625$ & $0.024 / 38070717$ & $0.000 / 33425.244$ \\
$H_{\mathrm{W} \text { (image) }}$ & 0.400 & 0.413 & 0.360 & 0.428 \\
$H_{\mathrm{W}(\mathrm{PDF})}$ & 0.560 & 0.565 & 0.426 & 0.689 \\
$H_{\mathrm{W} \text { (firmagram) }}$ & 0.427 & 0.319 & 0.466 & 0.497 \\
\hline
\end{tabular}

Table 6. Pearson-r correlation matrix for the standard deviations $(S)$ of three applied techniques: Box dimension, $R / S$ analysis and Power spectrum.

\begin{tabular}{|c|c|c|c|c|c|c|}
\hline & $S_{\text {box (image) }}$ & $S_{\text {box (firmagram) }}$ & $S_{\mathrm{RS} \text { (image) }}$ & $S_{\mathrm{RS}(\mathrm{PDF})}$ & $S_{\text {PS (image) }}$ & $S_{\mathrm{PS}(\mathrm{PDF})}$ \\
\hline \multirow{2}{*}{$S_{\text {box(image) }}$} & 1 & $-0.528^{*}$ & 0.059 & -0.013 & -0.207 & 0.179 \\
\hline & & 0.029 & 0.821 & 0.960 & 0.426 & 0.492 \\
\hline \multirow{2}{*}{$S_{\text {box(firmagram })}$} & & 1 & -0.249 & 0.286 & -0.128 & 0.126 \\
\hline & & & 0.336 & 0.266 & 0.625 & 0.629 \\
\hline \multirow[t]{2}{*}{$S_{\mathrm{RS} \text { (image) }}$} & & & 1 & 0.014 & $-0.715^{* *}$ & -0.321 \\
\hline & & & & 0.959 & 0.001 & 0.210 \\
\hline \multirow[t]{2}{*}{$S_{\mathrm{RS}(\mathrm{PDF})}$} & & & & 1 & -0.012 & 0.475 \\
\hline & & & & & 0.965 & 0.054 \\
\hline \multirow[t]{2}{*}{$S_{\text {PS(image) }}$} & & & & & 1 & 0.331 \\
\hline & & & & & & 0.194 \\
\hline$S_{\mathrm{PS}(\mathrm{PDF})}$ & & & & & & 1 \\
\hline
\end{tabular}

* Correlation is significant at the 0.05 level (2-tailed).

** Correlation is significant at the 0.01 level (2-tailed).

The mean $H_{\mathrm{RS}}$ value measured on the firmagram was equal to 0.229 (with standard deviation 0.141 ) showing that the $H_{\mathrm{RS}}$ extracted from the original image has higher roughness (mean $\left.H_{\mathrm{RS}}=0.044\right)$ and higher uncertainty $\left(S_{\mathrm{RS}}=\right.$ 0.493 ). The power spectrum technique gave the similar mean $H_{\mathrm{PS}}$ value of 0.208 for the original images of the compared soils, showing higher roughness for the firmagram $\left(H_{\mathrm{PS}}=\right.$ 0.048). The values of $H_{\mathrm{W}}$ measured by Wavelets method fluctuated around 0.5 for images, firmagrams and PDF, the mean $H_{\mathrm{W}}$ value was equal to 0.4 for the original images (Table 5).

This conclusion is confirmed by Pearson's $r$ analysis where 36 different pairs of $H$ were compared (Table 7), showing statistically significant correlation at the 0.01 level between 5 of them, at the level 0.05 between three pairs, and lack of correlation between the remaining 28 pairs.

In spite of the statistical similarity between the compared soils' roughness (Table 4), the precision of the ap- plied fractal techniques measured in terms of standard deviation was significantly different, except for the following pairs which show significant correlation: $S_{\text {box (image) versus }}$

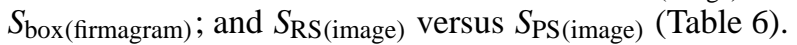

The outlier (high) standard deviation values of the spectral dimension might be due to the special construction of the time series extracted from the digital images and firmagrams. In the image one should expect a spatial correlation over a distance of a few pixel-sizes between the neighboring values $p_{i, j} ; p_{i \pm 1, j} ; p_{i \pm 2, j} ; \ldots$. Because of this, some artificial periodicity in the firmagram of period $\approx N$ could have appeared, so that the lags, window-length, etc. used to estimate $H$ or $D$ from the images and firmagrams must be kept much less than image size $N$ in order to avoid this artifact.

Statistical comparison was also accomplished among the central moments (mean, variance, kurtosis, skewness) of the empirical and theoretical PDFs extracted by the @ Risk software, confirming the similarity of the gray-level 
Table 7. Pearson-r correlation matrix for the Hurst exponents of four techniques applied: Box dimension, $R / S$ analysis, Power spectrum and Wavelets.

\begin{tabular}{|c|c|c|c|c|c|c|c|c|c|}
\hline & $H_{\text {box (image) }}$ & $H_{\text {box (firmagram) }}$ & $H_{\mathrm{RS} \text { (image) }}$ & $H_{\mathrm{RS}(\mathrm{PDF})}$ & $H_{\mathrm{PS} \text { (image) }}$ & $H_{\mathrm{PS}(\mathrm{PDF})}$ & $H_{\mathrm{W} \text { (image) }}$ & $H_{\mathrm{W}(\mathrm{PDF})}$ & $H_{\mathrm{W} \text { (firmagram) }}$ \\
\hline \multirow{2}{*}{$H_{\text {box (image) }}$} & 1 & -0.341 & -0.405 & -0.436 & 0.446 & -0.414 & 0.213 & -0.114 & 0.094 \\
\hline & & 0.181 & 0.107 & 0.081 & 0.073 & 0.099 & 0.412 & 0.664 & 0.719 \\
\hline \multirow[t]{2}{*}{$H_{\text {box(firmagram) }}$} & & 1 & $0.748^{* *}$ & $0.718^{* *}$ & $-0.869^{* *}$ & 0.457 & -0.01 & -0.175 & 0.328 \\
\hline & & & 0.001 & 0.001 & 0.000 & 0.065 & 0.969 & 0.501 & 0.199 \\
\hline \multirow[t]{2}{*}{$H_{\mathrm{RS} \text { (image) }}$} & & & 1 & $0.583^{*}$ & $-0.674^{* *}$ & $0.608^{*}$ & -0.149 & -0.007 & 0.004 \\
\hline & & & & 0.014 & 0.003 & 0.010 & 0.569 & 0.979 & 0.987 \\
\hline$H_{\mathrm{RS}(\mathrm{PDF})}$ & & & & 1 & $-0.582^{*}$ & $0.644^{* *}$ & -0.132 & 0.022 & 0.463 \\
\hline$H_{\mathrm{PS} \text { (image) }}$ & & & & & & 0.100 & 0.466 & 0.932 & 0.340 \\
\hline \multirow[t]{2}{*}{$H_{\mathrm{PS}(\mathrm{PDF})}$} & & & & & & 1 & -0.391 & 0.130 & -0.128 \\
\hline & & & & & & & 0.121 & 0.620 & 0.624 \\
\hline \multirow[t]{2}{*}{$H_{\mathrm{W} \text { (image) }}$} & & & & & & & 1 & 0.140 & 0.133 \\
\hline & & & & & & & & 0.591 & 0.610 \\
\hline$H_{\mathrm{W}(\mathrm{PDF})}$ & & & & & & & & 1 & 0.057 \\
\hline
\end{tabular}

* Correlation is significant at the 0.05 level (2-tailed).

** Correlation is significant at the 0.01 level (2-tailed).

distribution across the Chernozem-Solonetz pedological complex images and the Chocolate Clay. We speculate that the influence of microstructure and soil mineralogy on image roughness is more important than that of all other attributes characteristic for the macrofeatures of the compared structural patterns. The detailed architecture of Solonetz on the microscale preserved the original Chernozem features, conserving the self-organizing capacity of the fine matrix near the transition point when sodium content overpasses the critical value. This catastrophic event, known as soil chemical degradation, involves a structural phase transition detectable by visual comparison of microscopic images through the pore pattern's changes but not measurable in terms of the Hurst exponent. The Chernozem aggregates collapsed at all hierarchical levels, resulting in the massive pattern of Solonetz, where the major attraction between the solid particles (with high content of montmorillonites) is responsible for the low inter-aggregate porosity and high fracture density. This re-arrangement of structural patterns does not cause statistically significant changes in the scale-invariance of the microstructure. However, in spite of the statistically insignificant differences of the Hurst exponent values, the $\mathrm{H}$ values of Solonetz and Chocolate Clay for some techniques tend to be smaller, indicating the tendency to higher roughness in comparison with Chernozem (Table 5). These changes can be interpreted as indications of the breakdown of the Chernozem's interconnected porosity in Solonetz, due to the soil's chemical degradation. In most of the analyzed cases the roughness of the micromorphological images has antipersistent character.

\section{Conclusions}

We propose the Fractal Metrology in order to describe the phase transition in a complex system and documented the ability of its toolbox to extract the qualitative and quantitative information about the spontaneous emergence of the massive structural pattern of Solonetz from the perfect spongy structure of Chernozem, as the sodium concentration inside the Cation Exchange Complex (CEC) exceeds a certain critical value. The multiscale information was extracted from the micromorphological digital images of these soils with contrasting structural patterns but similar mineralogy (Chernozem, Solonetz and Chocolate Clay). Two of them belong to the same pedological complex affected by a chemical degradation (salinization) accompanying by the destruction of soil aggregates. In spite of drastic visual changes in the structural features of solid/pore patterns, the roughness of the soil's digital images, measured in terms of Hurst exponent by selected fractal techniques, were statistically similar. Notwithstanding, the three main Fractal Metrology techniques, proposed by us, were able to extract the multiscale tendencies in the soil's structural dynamics on global (roughness of the probability density function of gray intensities) and local scales (firmagrams and digital images). Chernozem has the highest roughness on the scale of macroaggregates (structural fractal) and the lowest on the pixel scale (textural fractal) related with the higher porosity and lower density of this perfectly aggregated soil. These results provide the suitability of the proposed step-by-step metrological procedure to describe qualitatively (visually and in terms of tendencies 
of roughness dynamics) and quantitatively the critical behavior of soil structural patterns during degradation. The fractal measurands extracted from the original digital images, firmagrams and probability density functions show different details of soil structural patterns while the Hurst exponent values were statistically similar for the case of phase transition. The box counting dimension extracted from the images and firmagrams was more precise in terms of standard deviation in comparison with the three other tested fractal techniques. The selected toolbox can be useful for the quantification of the spatio-temporal dynamics and behavior of other complex Earth systems, especially in similar cases of phase-transition between order and disorder. The proposed toolbox is still far from being complete, most importantly standardized measures of image lacunarity are to be found in order to keep track of the soils' translation invariance during phase transition.

Acknowledgements. This research has been funded by Consejo de la Ciencia y Tecnología (CONACyT) México, under Grant 84502 and PAPIIT (UNAM) IN116009. The first author acknowledges the postgraduate scholarship support of CONACyT. G. Korvin gratefully acknowledges the creative atmosphere of his home institute, KFUPM, and the many visits to Mexico when he has had opportunity to interact with Oleschko's research group.

Edited by: S. De Bartolo

\section{References}

Alvarez-Ramirez, J., Echeverria, J. C., and Rodriguez, E.: Performance of a high-dimensional $R / S$ method for Hurst exponent estimation, Physica A, 387, 6452-6462, 2008.

Avnir, D. and Farin, D.: Molecular fractal surfaces, Nature, 308, 261-263, 1984.

Bakucz, P. and Krüger-Sehm, R.: A new wavelet filtering for analysis of fractal engineering surfaces, Wear, 266, 539-542, 2009.

Barton, C. C. and La Pointe, P. R. (Eds.): Fractals in Petroleum Geology and Earth Processes, Springer New York, 342 pp., 1995.

Bassler, K. E., Gunaratne, G. H., and McCauley, J. L.: Markov processes, Hurst exponents, and nonlinear diffusion equations: With application to finance, Physica A, 369, 343-353, 2006.

Behery, G. M.: Simulation of fractal dimension evaluations, Int. J. Model. Simul., 26, 91-97, 2006.

Beiró, M. G., Alvarez-Hamelin, J. I., and Busch, J. R.: A low complexity visualization tool that helps to perform complex systems analysis, New J. Phys., 10, 125003, doi:10.1088/13672630/10/12/125003, 2008.

Bigerelle, M., Gautier, A., and Iost, A.: Roughness characteristic length scales of micro-machined surfaces: A multi-scale modeling, Sensor Actuat. B-Chem., 126, 126-137, 2007.

Borri-Brunetto, M., Carpinteri A., and Chiaia B.: Scaling phenomena due to fractal contact in concrete and rock fractures, Int. J. Fracture, 95, 221-238, 1999.

Brewer, R.: Fabric and Mineral Analysis of Soils, John Willey and Sons, New York, 470 pp., 1964.
Brink, L.: The Nobel Prize in Physics 2008, Presentation Speech, http://nobelprize.org/nobel_prizes/physics/laureates/2008/ presentation-speech.html, 2008.

Chandrasekaran, S. and Sundararajan, S.: Effect of microfabrication processes on surface roughness parameters of silicon surfaces, Surf. Coat. Tech., 188-189, 581-587, 2004.

Chang, K. J., Ray, G., Bradbury, D., Nakagawa, O. S., Oh, S.-Y., Bartelink, D., Chang, E., Stine, B., Maung, T., Divecha, R., Boning, D., and Chung, J.: Using a Statistical Metrology Framework to Identify Systematic and Random Sources of Die- and Waferlevel ILD Thickness Variation in CMP Processes, IEDM Tech. Digest, 499-502, 1995.

Christensen, K. and Moloney, N. R.: Complexity and Criticality, Imperial College Press Advanced Physics Texts, Vol. 1, London, 392 pp., 2005.

Cox, M. G., Rossi, G. B., Harris, P. M., and Forbes, A.: A probabilistic approach to the analysis of measurement processes, Metrologia, 45, 493-502, 2008.

Cox, S.: Information Technology: the global key to precision agriculture and sustainability, Comput. Electron. Agr., 36, 93-111, 2002.

Curtis, M. and Farago, F.: Handbook of Dimensional Measurement, fourth edition, Industrial Press Inc., New York, USA, 608 pp., 2007.

Dathe, A., Eins, S., Niemeyer, J., and Gerold, G.: The surface fractal dimension of the soil-pore interface as measured by image analysis, Geoderma, 103, 203-229, 2001.

Denisov, S. I.: Fractal dimension of random processes, Chaos Soliton. Fract., 9, 1491-1496, 1998.

Diehl, R. J. and Holm, P.: Roughness measurements - Have the necessities changed? J. Sound Vib., 293, 777-783, 2006.

Dimri, V. P. and Prakash, M. R.: Scaling of power spectrum of extinction events in the fossil record, Earth Planet. Sc. Lett., 186, 363-370, 2001.

Dodds, P. S.: Optimal form of branching supply and collection networks, Phys. Rev. Lett., 104, 048702, doi:10.1103/PhysRevLett.104.048702, 2010.

Eberhardt, K., Hagwood, C., Kacker, R., Levenson, M., Liu, H. K., Vangel, M., Yen, J., and Zhang, N. F.: 5. 6 Bayesian Metrology, in: Report of activities of the statistical engineering division, NIST, edited by: Eberhardt, K., available at: http://www.itl.nist. gov/div898/pubs/ar/ar2000/node40.html, 2001.

Ellis, C.: The sampling properties of Hurst exponent estimates, Physica A, 375, 159-173, 2007.

El-Sonbaty, I. A., Khashaba, U. A., Selmy, A. I., and Ali, A. I.: Prediction of surface roughness profiles for milled surfaces using an artificial neural network and fractal geometry approach, J. Mater. Process. Tech., 200, 271-278, 2008.

Falconer, K.: Techniques in Fractal Geometry, John Wiley \& Sons, 274 pp., 1997.

Feagin, R. A.: Relationship of second order lacunarity, Brownian motion, Hurst exponent, and pattern organization, Physica A, 328, 315-321, 2003.

Feagin, R. A., Wu, X. B., and Feagin, T.: Edge effects in lacunarity analysis, Ecol. Model., 201, 262-268, 2007.

Fleming, R. W., Dror, R. O., and Adelson, E. H.: Real-world illumination and the perception of surface reflectance properties, J. Vision, 3, 347-368, 2003.

Fransson, J. H. M., Talamelli, A., Brandt, L., and Cusso, C.: 
Delaying transition to turbulence by a passive mechanism, Phys. Rev. Lett., 96(6), 064501, doi:10.1103/PhysRevLett.96.064501, 2006.

Gallant, J. C., Moore, I. D., and Hutchinson, M. F.: Estimating fractal dimension of profiles: a comparison of methods, Math. Geol., 26, 455-481, 1994.

Germer, T. A.: Measurement of roughness of two interfaces of a dielectric film by scattering ellipsometry, Phys. Rev. Lett., 85, 349-352, 2000.

Gomez, S., Hale, K., Burrows, J., and Griffiths, B.: Measurements of surface defects on optical components, Meas. Sci. Technol., 9, 607-616, 1998.

Hardy, G. H.: Weierstrass's non-diferentiable function, T. Am. Math. Soc., 17, 301-325, 1916.

Heilmann, R. K., Chen, C. G., Konkola, P. T., and Schattenburg, M. L.: Dimensional Metrology for Nanometer-Scale Science and Engineering: towards sub-nanometer accurate encoders, Nanotechnology, 15, 504-511, 2004.

Hopp, T. H.: Computational Metrology, Manufacturing Review 6, American Society of Mechanical Engineers, New York, 295304, 1993.

Hurst, H. E.: Long-term storage capacity of reservoirs, T. Am. Soc. Civ. Eng., 116, 770-808, 1951.

International Organization for Standardization (ISO): International Vocabulary of Basic and General Terms in Metrology, 3rd edition, Geneva, Switzerland, 55 pp., 2004.

Jensen, J. E.: Computationally modeling the effects of surface roughness on soft X-ray multilayer reflectors, M.S. thesis, Department of Physics and Astronomy, Brigham Young University, 164 pp., 2006.

Joint Committee for Guides in Metrology (JCGM): International Vocabulary of Metrology - Basic and general concepts and associated terms, 3rd edition, 90 pp., 2008.

Joint Committee for Guides in Metrology (JCGM): Evaluation of measurement data - An introduction to the "Guide to the expression of uncertainty in measurement" and related documents, 1 st edition, 20 pp., 2009.

Jones, C. L. and Jelinek, H. F.: Wavelet Packet Fractal Analysis of Neuronal Morphology, Methods, 24, 347-358, 2001.

Katz, J. S.: Indicators for complex innovation systems, Res. Policy, 35, 893-909, 2006.

Kim, T. W., Bhushan, B., and Cho, Y. J.: The contact behavior of elastic/plastic non-Gaussian rough surfaces, Tribol. Lett., 22, 113, 2006.

Klinkenberg, B.: A review of methods used to determine the fractal dimension of linear features, Math. Geol., 26, 23-46, 1994.

Kogan, J.: Introduction to clustering large and high dimensional data, Cambridge University Press, New York, 222 pp., 2007.

Korvin, G.: Fractal models in the earth sciences, Elsevier, Amsterdam, 396 pp., 1992.

Korvin, G.: Is the optical image of a non-Lambertian fractal surface fractal?, IEEE Geosci. Remote S., 2, 380-383, 2005.

Levitz, P.: Toolbox for 3-D imaging and modeling of porous media: Relationship with transport properties, Cement Concrete Res., 37, 351-359, 2007.

Lojkowski, W., Turan, R., Proykova, A., and Daniszewska, A. (Eds.): Eight Nanoforum Report: Nanometrology, available at: http://www.innovationsgesellschaft.ch/images/fremde publikationen/NANOMETROLOGY_Report.pdf, 2006.
Lu, X. and Guan, J.: A new approach to building histogram for selectivity estimation in query processing optimization, Comput. Math. Appl., 57, 1037-1047, 2009.

Majumdar, A. and Bhushan, B.: Fractal model of elastic-plastic contact between rough surfaces, ASME J. Tribol., 113, 1-11, 1991.

Malamud, B. D. and Turcote, D. L.: Self-affine time series: measures of weak and strong persistence, J. Stat. Plan. Infer., 80, 173-196, 1999.

Mandelbrot, B. B.: The fractal geometry of nature, edited by: Freeman, W. H. and Company, New York, USA, 1982.

Mandelbrot, B. B.: Gaussian Self-Affinity and fractals, SpringerVerlag, New York, 654 pp., 2002.

Mandelbrot, B. B. and Van Ness, J. W.: Fractional Brownian motions, fractional noises and applications, SIAM Rev., 10, 422437, 1968.

Mandelbrot, B. B. and Wallis, J. R.: Noah, Joseph and Operational Hydrology, Water Resour. Res., 4, 909-918, 1968.

Mandelbrot, B. B., Passoja, D. E., and Paulay, A. J.: Fractal character of fracture surfaces of metals, Nature, 308, 721-722, 1984.

Marschal, A., Andrieux, T., Compagon, P. A., and Fabre, H.: Chemical metrology - QUID?, Accredit. Qual. Assur., 7, 42-49, 2002.

MINITAB for Windows: MAVV. Home. WIN 1221.03737., available at: http://www.minitab.com, 1998.

Monroe, D.: Rough skin is good, Phys. Rev. Focus, available at: http://focus.aps.org/story/v17/st6, 2006.

NIST Bayesian metrology, National Institute of Standards and Technology: Manufacturing Engineering Laboratory, http://www.itl.nist.gov/div898/bayesian/overview.html, access: 9 November 2010, 2001.

NIST/SEMATECH: e-Handbook of statistical methods, Probability Density Function, http://www.itl.nist.gov/div898/handbook/, 2006.

Oleshko, K., Vadiunina, A. F., Zilaeva, V. A., and Truchin, V. I.: Influencia del campo magnético sobre las propiedades del suelo y plantas, Pochvovedenie, 7, 91-100, 1980 (in Russian).

Oleschko, K.: Effect of electric current on clay minerals in "chocolate" clays, Universidad Estatal de Moscú M.V. Lomonósov, Facultad de Suelos, Boletín de la Universidad de Moscú, serie Pochvovedenie (Edafología), No. 2, 1981 (in Russian).

Oleschko, K., Figueroa, B., Miranda, M. E., Vuelvas, M. A., and Solleiro R. E.: Mass fractal dimensions and some selected physical properties of contrasting soils and sediments of México, Soil Till. Res., 55, 43-61, 2000.

Oleschko, K., Korvin, G., Balankin, A. S., Khachaturov, R. V., Flores, Figueroa, L., Urrutia, J., and Brambila, F.: Fractal Scattering of Microwaves from Soils, Phys. Rev. Lett., 89, 188501, doi:10.1103/PhysRevLett.89.188501, 2002.

Oleschko, K., Korvin, G., Figueroa, B., Vuelvas, M. A., Balankin, A., Flores, L., and Carreon, D.: Fractal radar scattering from soil, Phys. Rev. E, 67, 041403-1:041403-13, doi:10.1103/PhysRevE.67.041403, 2003.

Oleschko, K., Parrot, J.-F., Ronquillo, G., Shoba, S., Stoops, G., and Marcelino, V.: Weathering: toward a fractal quantifying, Math. Geol., 36(5), 607-627, 2004.

Oleschko, K., Parrot, J.-F., Korvin, G., Esteves, M., Vauclim, M., Torres-Argüelles, V., Gaona-Salado, C., and Cherkasov, S.: Fractal Image Informatics: from SEM to DEM, Proceedings of 4th International Conference: GIS in Geology and Geosciences, 
Vista of new approaches for the Geoinformatics, AIP, 109, 25092517, 2008.

Oleschko, K., Korvin, G., Flores, L., Brambila, F., Gaona, C., Parrot, J.-F., Ronquillo, G., and Zamora, S.: Probability density function: A tool for simultaneous monitoring of pore/solid roughness and moisture content, Geoderma, doi:10.1016/j.geoderma.2009.10.015, in press, 2010.

Orbach, R.: Dynamics of fractal networks, Science, 231, 814-819, 1986.

Palisade Corporation: Guide to Using @ RISK: Risk Analysis and Simulation Add-In for Microsoft ${ }^{\circledR}$ Excel Version 5.5, 2005.

Parfenova, E. I. and Yarilova, E. A.: Guide on Micromorphological Studies in Soil Science, Nauka, Moscow, 198 pp., 1977 (in Russian).

Partis, L., Croan, D., King, B., and Emslie, K. R.: Biometrology and biomeasurement: overview of current technologies and priorities for a biological measurement program in Australia, Australian Government Analytical Laboratories, Pymble, NSW, Australia, AGAL Public Interest Program Public, Interest Report Series Number, 2002-1, 2002.

Paschotta, R.: Encyclopedia of laser physics and technology, RP Photonics, Wiley-VCH, Berlin, 844 pp., 2009.

Pawley, J. B. (Ed.): Handbook of biological confocal microscopy, 3rd edition, Springer Science + Business media, New York, 985 pp., 2006.

Pelletier, J. D. and Turcotte, D. L.: Self-affine time series: 11. Applications and models, Adv. Geophys., 40, 91-166, 1999.

Pendleton, D. E., Dathe, A., and Baveye, P.: Influence of image resolution and evaluation algorithm on estimates of the lacunarity of porous media, Phys. Rev. E, 72, 041306, doi:10.1103/PhysRevE.72.041306, 2005.

Pentland, A. P.: Fractal based description of natural scenes, IEEE T. Pattern Anal., PAMI-6, 661-674, 1984.

Plowman, D. A., Solansky, S., Beck, T. E., Baker, L., Kulkarni, M., and Villarreal Travis, D.: The role of leadership in emergent, self-organization, The Leadership Quarterly, 18, 341-356, 2007.

Puente, C.: A universe of projections: may Plato be right?, Chaos Soliton. Fract., 19, 241-253, 2004.

Rehman, S. and Siddiqi, A. H.: Wavelet based Hurst exponent and fractal dimensional analysis of Saudi climatic dynamics, Chaos Soliton. Fract., 40, 1081-1090, 2009.

Rodriguez-Iturbe, I. and Rinaldo, A.: Fractal river basins: chance and self-organization, Cambridge University Press, New York, 564 pp., 1997.

Scipioni, A., Rischette, P., Bonhomme, G., and Devynck, P.: Characterization of self-similarity properties of turbulence in magnetized plasmas, Phys. Plasmas, 15, 112303, doi:10.1063/1.3006075, 2008.

Seffens, W.: Order from chaos, Techsighting Software, Science, 285, 5431, doi:10.1126/science.285.5431.1228a, 1999.

SML, Surface Metrology Laboratory: Departament of Mechanical Engineering, Worcester Polytechnic Institute, 2008.
Stanley, H. E.: Introduction to Phase Transitions and Critical Phenomena, Oxford University Press, 336 pp., 1971.

Statistical Package for the Social Sciences (SPSS), 13.0 for Windows, Copyright (C) SPSS Inc., released on 6 December 2004.

Strauss, O.: Quasi-continuous histograms, Fuzzy Set. Syst., 160, 2442-2465, 2009.

$\mathrm{Su}, \mathrm{Z} .-\mathrm{Y}$. and $\mathrm{Wu}, \mathrm{T}$.: Music walk, fractal geometry in music, Physica A, 380, 418-428, 2007.

Sun, W., Xu, G., Gong, P., and Liang, S.: Fractal analysis of remotely sensed images: a review of methods and applications, Int. J. Remote Sens., 27, 4963-4990, 2006.

Tancrez, J.-S., Semal, P., and Chevalier, P.: Histogram based bounds and approximations for production lines, Eur. J. Oper. Res., 197, 1133-1141, 2009.

Tang, D. and Marangoni A. G.: 3-D fractal dimension of fat crystal networks, Chem. Phys. Lett., 433, 248-252, 2006.

Tél, T. and Gruiz, M.: Chaotic Dynamics: An Introduction Based on Classical Mechanics, Cambridge University Press, 393 pp., 2006.

TruSoft, Intern. Inc.: Benoit 1.3 Software, 1999.

Turcotte, D. L.: Fractals and Chaos in Geology and Geophysics, Cambridge University Press, 416 pp., 1997.

Vadyunina, A. F., Sokolova, T. A., Oleshko, K., and El Lakani, A. A.: Effect of electric current on clay minerals in "Chocolate Clays”, Vestnik MGU, 50-54, 1980 (Pochvovedenie, in Russian).

Van Gorp, A., Bigerelle, M., Grellier, A., Iost, A., and Najjar D.: A multi-scale approach of roughness measurements: Evaluation of the relevant scale, Mat. Sci. Eng. C, 27, 1434-1438, 2007.

Verhoest, N. E. C., Lievens, H., Wagner, W., Álvarez-Mozos, J., Moran, M. S., and Mattia, F.: On the soil roughness parameterization problem in soil moisture retrieval of bare surfaces from synthetic aperture radar (Review), Sensors, 8, 4213-4248, 2008.

Villarrubia, J. S.: Issues in line edge and line width roughness metrology, AIP Conference proceedings: Characterization and metrology for ULSI Technology, 386-393, 2005.

Wang, L., Rong, W., and Sun, L.: Elastic-plastic adhesive contact of fractal microparts surfaces with low adhesion parameters, Proceedings of the 3rd IEEE Int. Conf. on Nano/Micro Engineered and Molecular Systems, Sanya, China, 6-9 January, 592-595, 2008.

Weisstein, E. W.: Fourier Transform, From Mathworld A Wolfram Web Resources, http://mathworld.wolfram.com/ FourierTransform.html, 2010

Willink, R.: Principles of probability and statistics for metrology, Metrologia, 43, S211-S219, 2005.

Wilson, T. H.: Short notice, Fractal strain distribution and its implications for cross-section balancing: further discussion, J. Struct. Geol., 19, 129-132, 1997. 